\title{
Association between periodontal disease and bone mineral density in postmenopausal women: A cross sectional study
}

\author{
Nishat Sultan ${ }^{1}$, Jyoti Rao ${ }^{2}$ \\ ${ }^{1}$ M.D.S., B.D.S, Senior Resident, Department of Periodontics, Centre for Dental Education and Research, All India Institute of \\ Medical Sciences, New Delhi, India \\ ${ }^{2}$ M.D.S., B.D.S, Professor and Head of the department, Department of Periodontics, Goa Dental College and Hospital, Goa, \\ India
}

Correspondence:

G.P.O. BOX NO. 1757.

DELHI-110006, INDIA.

nishat_sultan@rediffmail.com

Received: $24 / 06 / 2010$

Accepted: $14 / 11 / 2010$

Sultan N, Rao J. Association between periodontal disease and bone mineral density in postmenopausal women: A cross sectional study. Med Oral Patol Oral Cir Bucal. 2011 May 1;16 (3):e440-7.

http://www.medicinaoral.com/medoralfree 01/v16i3/medoralv16i3p440.pdf

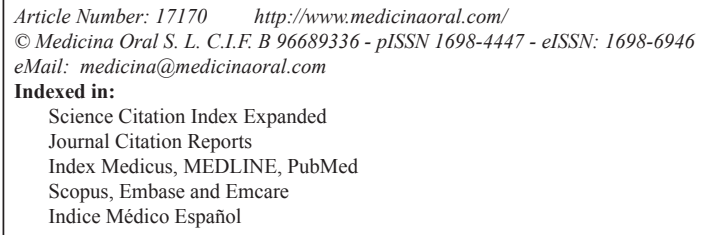

\begin{abstract}
Objectives: Both periodontitis and osteoporosis represent major health problems especially in elderly women. The relationship between the two diseases and oral bone loss is important having significant public health impact in the prevention of morbidity and mortality related to these disorders. The present study was aimed to investigate the possible association between osteoporosis and periodontal disease among postmenopausal women residing in Goa, India. Study design: A complete periodontal examination (all teeth except third molar) including plaque index (PI), gingival index (GI), clinical attachment loss (CAL) measurement was performed on 80 dentate Goan postmenopausal women (age $\geq 50 \mathrm{yrs}$ ) with generalized chronic periodontitis. Mean alveolar bone loss (ABL) was measured from full mouth intraoral periapical radiographs, by recording the distance from cementoenamel junction (CEJ) and the most coronal portion of alveolar crest at mesial and distal aspect of all teeth except canines and third molars. Systemic bone loss was determined from hand-wrist radiograph of the patient through Digital X-Ray Radiogrammetry. Statistical analysis was done to assess the relationships between periodontal variables and bone mineral density (BMD) after adjusting for age, years since menopause, body mass index (BMI), smoking, number of remaining teeth, PI and GI. Results: Age of the patient, years since menopause and BMI showed significant correlation with BMD. CAL and ABL showed mildly negative and statistically nonsignificant correlation with the BMD. Of all the variables studied, only smoking and BMI were strong predictors of BMD. Conclusion: Skeletal BMD is related to interproximal ABL and CAL, though not to a statistically significant level; implicating postmenopausal osteopenia as a risk indicator for periodontal disease.
\end{abstract}

Key words: Periodontal disease, osteoporosis, osteopenia, postmenopausal women, bone mineral density, risk factors. 


\section{Introduction}

Both periodontitis and osteoporosis represent major health problems, with increased incidence and severity with advancing age, all over the world. A Consensus Development Conference (2001) defined osteoporosis as "a systemic skeletal disease characterized by low bone mass and microarchitectural deterioration with consequent increase in bone fragility and susceptibility to fracture" (1). According to the World Health Organization, osteoporosis is considered to be present when bone mineral density (BMD) is 2.5 standard deviations (SD) below the young normal. Osteopenia is defined as bone density levels between $1 \mathrm{SD}$ and $2.5 \mathrm{SD}$ below normal BMD (2). Loss of bone mass, per se, does not cause symptoms. However, once a fracture does occur, pain, loss of function, and, in some cases, deformity may result. For these reasons, osteoporosis before fractures is termed a "silent disease".

Periodontitis is an inflammatory disease characterized by loss of connective tissue and alveolar bone. Like osteoporosis, it is a silent disease, not causing symptoms until late in the disease process, such as mobile teeth, abscesses, and tooth loss. Both periodontitis and osteoporosis are bone-resorptive diseases.

Osteoporosis, though not being the initial cause of periodontitis, has been shown to be a risk indicator that may contribute to the progression of periodontal disease. Although some studies that attempted to correlate osteoporosis with periodontal disease obtained significant results (3-6), others, did not $(7,8)$. With this perspective, the present study was aimed to investigate the possible association between osteoporosis and periodontal disease among postmenopausal Goan women.

\section{Materials and Methods}

1. This cross-sectional study consisted of 80 dentate Goan postmenopausal women (age $\geq 50 \mathrm{yrs}$ ) with generalized chronic periodontitis visiting Periodontics clinic, Goa Dental College and Hospital, Goa. Postmenopausal women ( $\geq 50 \mathrm{yrs}$ ) having generalized chronic periodontitis (generalized clinical attachment loss $\geq 1 \mathrm{~mm}$ and bleeding on probing) with at least 10 natural teeth present excluding the $3^{\text {rd }}$ molars were included. Severity of periodontal disease, i.e., mild, moderate and severe was not kept as the selection criteria so as to study the relation between the range of different levels of periodontal disease and BMD.

2. Exclusion criteria were: patients requiring antibiotic prophylaxis; those having parathyroid and metabolic bone disease, cancer, or on long term steroid therapy; having early onset of menopause; having history of hysterectomy; and alcoholics. All the participants signed the written informed consent.

3. Questionnaires were filled for each patient, eliciting information on medical and dental history, age at meno- pause, years since menopause, smoking status, history of estrogen supplementation and whether previously diagnosed of osteoporosis.

4. Complete periodontal examination was performed on all teeth except 3rd molars that included: 1) Plaque index (PI) (9), 2) Gingival index (GI) (10), 3) Probing depth measurement, 4) Clinical attachment loss (CAL) measurement using Williams periodontal probe (HuFriedy ${ }^{\mathrm{TM}}$ ), at four sites on each tooth: mesiobuccal, midbuccal, distobuccal and midlingual.

5. Alveolar bone loss (ABL) was determined from full mouth IOPA radiographs through paralleling radiographic technique as the distance from cementoenamel junction (CEJ) and the most coronal portion of alveolar crest on all the teeth except $3^{\text {rd }}$ molars and canines. All the study radiographs were analyzed using a digital method. Individual X-rays were scanned using HewlettPackard Scanjet Scanner 2400 series, measurements were carried out using computer software program $(A u$ toCAD-2006) and the mean was calculated (Fig. 1).

6. Systemic bone loss was determined from hand-wrist radiograph of the patient using Digital X-ray Radiogrammetry (DXR), (Pronosco X-posure system ${ }^{\mathrm{TM}}$ ).

Each radiograph covers the non-dominant hand and forearm. The radiographs were obtained using an $18 \times 43$ Imation green-sensitive, high contrast mammography film with a relative speed of 100 . The hand and forearm were placed flat on the cassette with the palm side facing the X-ray cassette. The centre of the X-ray beam was placed approximately $2 \mathrm{~cm}$ above the wrist. A filmfocus distance of $102 \mathrm{~cm}$ was used with an exposure of $50 \mathrm{kV}$ and 4-6 mAs. The effective radiation dose for the method was $1 \mu \mathrm{Sv}$, which is the radiation dose for a standard forearm radiograph.

After development, the radiographs were scanned and analyzed automatically using the Pronosco X-posure System $^{\mathrm{TM}}$. The software in the system derives the DXR BMD $\left(\mathrm{gcm}^{2}\right)$ through a completely automated analysis of the cortical thickness of the ulna, radius and the three middle metacarpal bones. After scanning of the radiograph, the program detects and analyses these bones without any guidance from the operator (Fig. 2).

-Statistical Analysis:

Statistical analysis was performed using statistical software (SPSS version 15). Descriptive statistics were calculated for all variables including means, standard deviations, ranges, and percentages. The relationships between periodontal variables with BMD were assessed by partial correlation coefficients (r) from multiple linear regression analysis adjusting for age, years since menopause, body mass index (BMI), smoking, number of remaining teeth, plaque index and gingival index. 


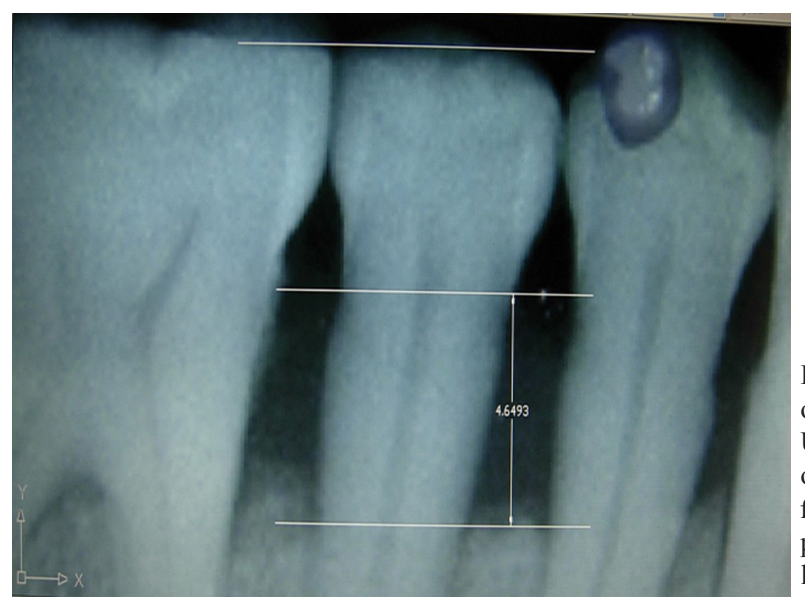

Fig. 1. Digital measurement of Radiographic Alveolar Crestal Bone Loss on mesial aspect of mandibular second premolar.

Using AutoCAD 2006 software a straight line was drawn at the level of occlusal plane, a second line was drawn at the level of CEJ parallel to the first one, a third line was drawn at the level of alveolar crest parallel to the previous two. The perpendicular distance between the second and third line represents the alveolar bone loss.

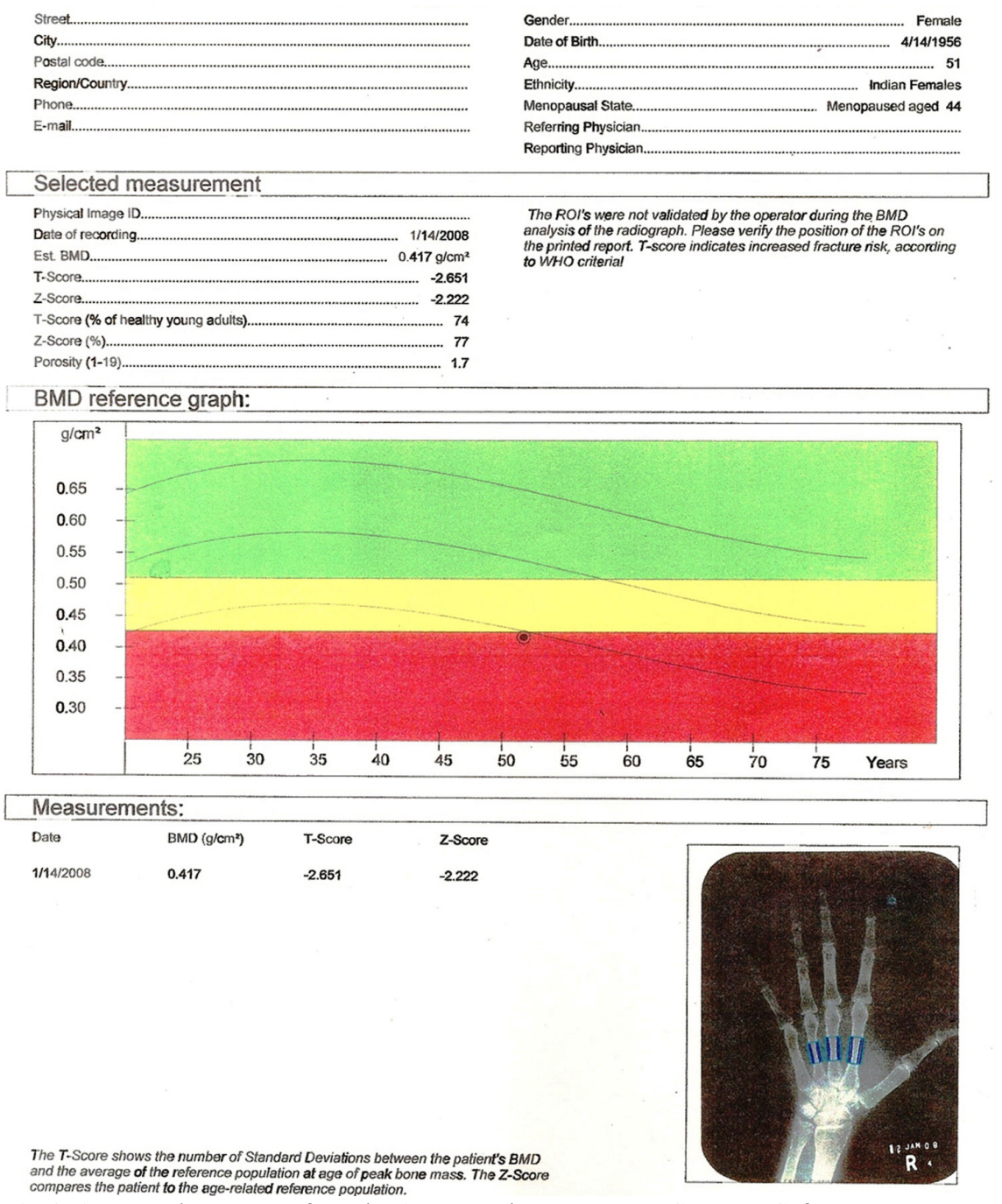

Fig. 2. An exemplary report of a patient's BMD using Pronosco ${ }^{\mathrm{TM}}$ Computer Software.

Once the basic details of the patient were entered, the Pronosco ${ }^{\mathrm{TM}}$ automatically calculates the BMD.

Bone density tests were classified into three diagnoses namely normal (green zone), osteopenia (yellow zone) and osteoporosis (red zone) and gives BMD value in $\mathrm{g} / \mathrm{cm}^{2}$. 


\section{Results}

In the present study sample $(\mathrm{n}=80)$, the mean age of the selected patients was $57.7 \mathrm{yrs}$, the mean menopausal age was $47.3 \mathrm{yrs}$ and the mean number of years since menopause was $10.3 y r s$. The mean BMI of the study sample was $25.8 \mathrm{~kg} / \mathrm{m}^{2}$. The mean CAL was $3.8 \pm 0.84 \mathrm{~mm}$ and the mean ABL was $4.2 \pm 0.84 \mathrm{~mm}$. The mean number of remaining teeth in the study population was 21.1.

The relationship between all the selected variables in the present study, with the skeletal bone mineral density as estimated by the hand-wrist radiograph was assessed by Pearson's correlation coefficients. The correlation coefficient, whether positive or negative, determines the degree or extent of association between the two variables. If large, it may be significant; otherwise it might have arisen by chance.

Age of the patient showed mildly negative correlation with BMD ( $\mathrm{r}=-0.381)$ and the association was highly significant, $(\mathrm{p}=0.000)$. Years since menopause also showed mildly negative correlation with the skeletal
BMD ( $r=-0.417)$ and the association was highly significant $(\mathrm{p}=0.000)$. Body mass index showed mildly positive correlation with the BMD $(r=0.263)$ and the association was statistically significant $(\mathrm{p}=0.018)$. The variables like age at menopause, plaque index, and the number of remaining teeth showed mildly positive correlation with the skeletal BMD but the association was not statistically significant $(\mathrm{p}>0.05)$. Clinical attachment loss and alveolar bone loss showed mildly negative correlation with the BMD, however, the association was not statistically significant ( $\mathrm{p}>0.05$ ) (Table 1 and Fig. 3 ).

Multiple regression analysis was done to predict the value of the dependent variable (BMD) after adjusting the independent variables (age, age at menopause, years since menopause, smoking, BMI, PI and GI). The results show that after univariate regression analysis, of all the variables studied, only age, body mass index and years since menopause affect BMD. After multivariate regression analysis, of all the variables, only BMI affects BMD. None of the periodontal factors affect BMD in multivariate regression analysis (Table 2).

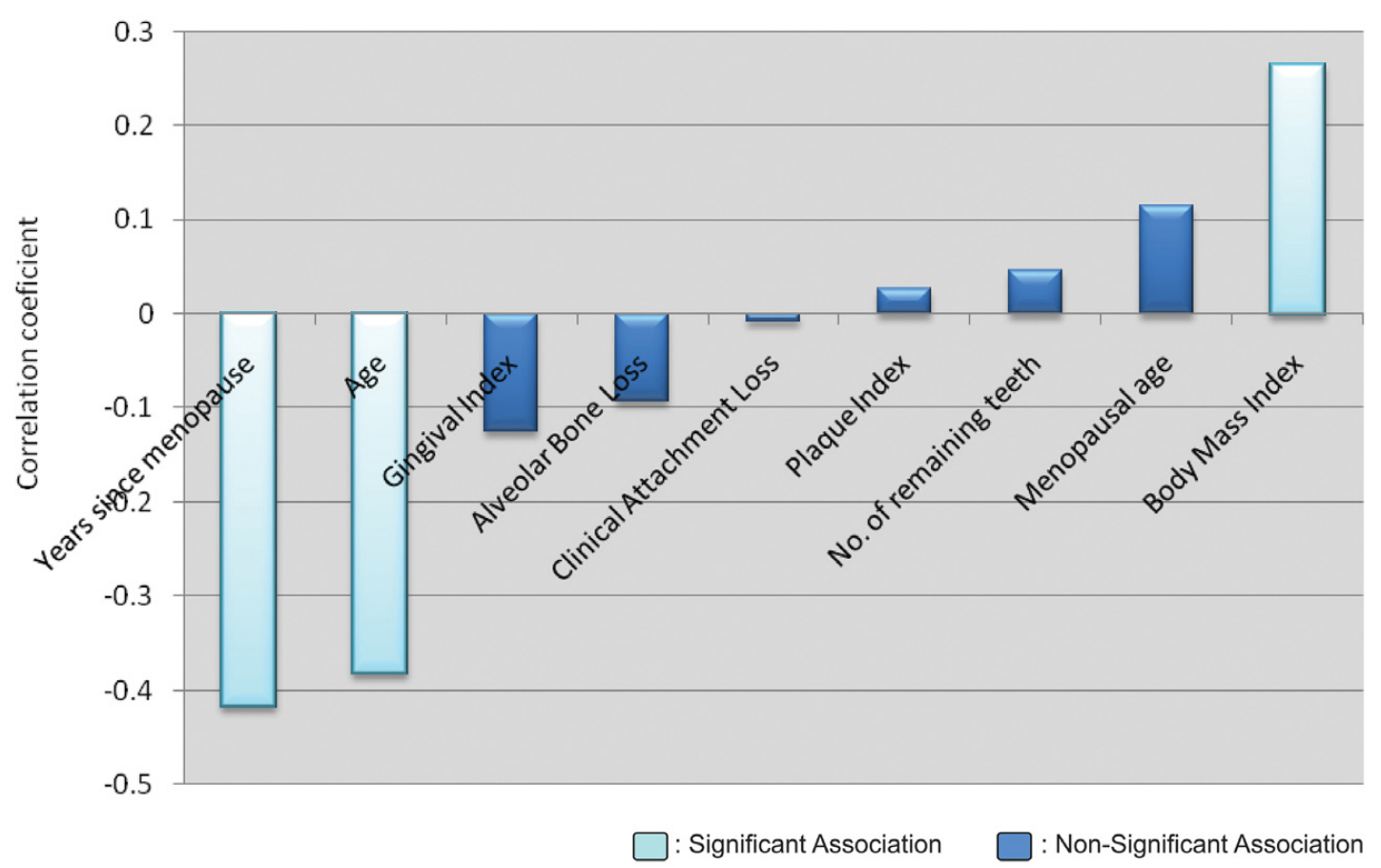

Fig. 3. Pearson's correlation coefficients between the study variables and the BMD.

Age of the patient and Years since menopause showed mildly negative and significant correlation with BMD, while BMI showed mildly positive and significant correlation with the BMD. CAL and ABL showed mildly negative but non-significant correlation with the BMD. 
Table 1. Description of the study population and Pearson's correlation coefficients among BMD and the study variables.

\begin{tabular}{|l|c|c|c|c|}
\hline \multicolumn{1}{|c|}{ Variables } & Mean & Standard Deviation & \multicolumn{2}{c|}{ Correlation with BMD } \\
\cline { 3 - 5 } & & & $\mathbf{r}^{\pi}$ & p-value \\
\hline BMD & 0.504 & 0.049 & $\ldots$ & $\ldots$ \\
\hline Age & 57.737 & 6.705 & -0.381 & 0.000 \\
\hline Menopausal Age & 47.325 & 3.507 & 0.116 & 0.307 \\
\hline Years since menopause & 10.387 & 7.050 & -0.417 & 0.000 \\
\hline Body mass index & 25.836 & 3.742 & 0.263 & 0.018 \\
\hline Plaque index & 1.851 & 0.190 & 0.027 & 0.814 \\
\hline Gingival index & 1.793 & 0.231 & -0.126 & 0.265 \\
\hline Clinical attachment loss & 3.833 & 0.846 & -0.009 & 0.937 \\
\hline Alveolar bone loss & 4.246 & 0.849 & -0.093 & 0.410 \\
\hline Number of remaining teeth & 21.087 & 4.736 & 0.047 & 0.047 \\
\hline
\end{tabular}

BMD: Bone mineral density

q: Pearson's correlation coefficient

Table 2. Univariate and multivariate regression analysis between the study variables and the BMD.

\begin{tabular}{|l|c|c|c|c|}
\hline \multirow{2}{*}{ Variables } & \multicolumn{2}{|c|}{ Unadjusted } & \multicolumn{2}{c|}{ Adjusted } \\
\cline { 2 - 5 } & $\boldsymbol{\beta ( S E )}$ & p-value & $\boldsymbol{\beta ( S E )}$ & p-value \\
\hline Age & $-0.002(0.001)$ & 0.000 & $-0.005(0.014)$ & 0.689 \\
\hline Menopausal Age & $0.001(0.001)$ & 0.307 & $0.004(0.014)$ & 0.733 \\
\hline Years since menopause & $-0.002(0.001)$ & 0.000 & $0.002(0.014)$ & 0.848 \\
\hline Body mass index & $0.003(0.001)$ & 0.018 & $0.003(0.001)$ & 0.013 \\
\hline Plaque index & $0.006(0.029)$ & 0.814 & $0.024(0.033)$ & 0.466 \\
\hline Gingival index & $-0.026(0.023)$ & 0.265 & $-0.033(0.027)$ & 0.216 \\
\hline Clinical attachment loss & $-0.001(0.006)$ & 0.937 & $0.009(0.009)$ & 0.314 \\
\hline Alveolar bone loss & $-0.005(0.006)$ & 0.410 & $-0.009(0.008)$ & 0.249 \\
\hline Number of remaining teeth & $0.000(0.001)$ & 0.682 & $0.001(0.001)$ & 0.383 \\
\hline
\end{tabular}

BMD: Bone mineral density

$\beta$ : Regression coefficient

SE: Standard Error 


\section{Discussion}

The present cross-sectional study was carried out to assess the relationship between periodontitis and systemic bone loss in postmenopausal Goan women. Subjects who were 50 years and above were selected for the study as it has been proved earlier that age-related loss of bone is more pronounced in women after the age of 50 years (11). All the subjects with a history of early menopause were excluded because early menopause i.e., $<40$ years without hormone replacement therapy predisposes to osteoporosis (12). A study population with a range of different levels of periodontal disease and BMD was intended, therefore, severity of these conditions was not a selection criterion. Systemic factors of bone remodeling may also modify local tissue response to periodontal infections, so all subjects having a history of metabolic bone disease, parathyroid disease, or, cancer were excluded from the study.

In women at menopause, $2-3 \%$ and $4-8 \%$ loss of cortical and trabecular bone occurs per year respectively. In the present study, age and years since menopause, showed mildly negative and statistically significant correlation with BMD ( $\mathrm{r}=-0.38 \&-0.41$, respectively; $\mathrm{p}<0.01)$. This can be explained by the fact that the first 5-10 years after menopause are associated with a period of estrogen depletion and consequent rapid bone loss; hence it is conceivable to find a significant association of age and year since menopause with BMD recently after or during this period of bone loss. In comparing the results of various studies, it is important to consider the age group of the study populations. Elders et al. (7) and Klemetti et al. (8) failed to show any association between periodontal disease and systemic bone loss, however, the age group of the study sample was relatively young (46$55 \mathrm{yrs}$ ) when it is not very likely to find severe signs of periodontitis or osteoporosis, if any.

Low body weight and weight loss are both established risk factors for low bone mass and for an increased rate of bone loss (13). In the present study, the body mass index showed mildly positive and significant correlation with the BMD $(r=0.263, p=0.018)$. The regression coefficient (b) between BMD and BMI was 0.0036, $\mathrm{p}=0.013$, the association being statistically significant. Thus $B M I$ is a strong predictor of estimating BMD. Plaque index and Gingival index showed mild but non-significant correlation with skeletal BMD $(r=0.027$ and -0.126 ; $\mathrm{p}=0.814$ and 0.265 respectively) and not strong predictors of $B M D$. This could be because oral hygiene status is dictated by many other factors like the dietary habits, socioeconomic and education status of an individual. In the present study, alveolar bone loss showed statistically non-significant and mild correlation with the BMD ( $\mathrm{r}=-$ 0.093, $\mathrm{p}=0.410$ ) and $\boldsymbol{A B L}$ is not a strong predictor of BMD $(\beta=-0.009, p=0.249)$.

In the present study BMD values were not significant- ly correlated with number of remaining teeth $(\mathrm{r}=0.04$, $\mathrm{p}=0.68$ ). Thus, number of remaining teeth is not a strong predictor of $\boldsymbol{B M D}(\beta=0.001, \mathrm{p}=0.383)$. A relatively younger mean age (57.7 years) of the study subjects may contribute to the lack of an association between tooth loss and BMD. Our results were consistent with the results of the studies by Elders et al. (7), Mohammad et al. (4), Weyant et al. (14), and Taguchi et al. (15), which failed to detect significant correlations between tooth loss and BMD. Elders et al. (7) assessed 286 female volunteers in the age group of 46 to 55 years of age and found that lumbar BMD or metacarpal cortical thickness (MCT) did not correlate with the number of missing teeth which could be due to the following three reasons: first, there was a selection bias, as the study subjects were volunteers, were periodontally and systemically healthy and not having severe periodontitis. Second, the age group of the study sample was relatively young (46-55), where there are chances that the severe manifestations of periodontitis and osteoporosis are not always likely to manifest. Third, the effect of confounding factors was not taken into account. Taguchi et al. (15) did not find any significant differences in the number of remaining teeth and BMD in subjects with periodontal symptoms. However, subjects without periodontal symptoms had significantly higher lumbar BMD. They suggested that a relatively younger mean age (56.6 years) of their subjects could have contributed to the lack of association between self-reported periodontal status and tooth loss. This was opposite to the findings by Kribbs (16) who reported greater tooth loss in females with diagnosed vertebral-compression fractures. Aström et al. (17) also reported that a low number of teeth can be used to predict hip fractures in elderly males and females. The contradictory findings between these studies (Kribbs (16) and Aström et al. (17)) could be due to the fact that in all these studies, confounding variables such as smoking were not adjusted.

However, the use of tooth loss as a surrogate for periodontal disease extent has several limitations. Tooth loss in many cases occurs for reasons unrelated to bone metabolism, such as caries or periodontal disease. Also the extent of disease around the remaining teeth is usually not taken into account.

In the present study smoking can be considered an independent and strong predictor of estimating BMD $(\beta=-0.097, p=0.033)$, but since only one subject in the present study was a smoker, the association cannot be clearly stated. In the present study, $\boldsymbol{C A L}$ is not a strong predictor of estimating $\boldsymbol{B M D}(\beta=0.009, \mathrm{p}=0.314)$, this could be due to the fact that as we do not have data on the premenopausal periodontal health of the women in our study, it is possible that the attachment loss occurred prior to menopause and thus cannot directly be correlated to the postmenopausal osteopenia. Some studies 
failed to find any correlation between CAL and BMD $(3,5,14,16,18)$, while some found positive association between the two $(19,20)$. Different study populations, sample sizes, and lack of consideration of confounding variables and effect modifiers explain some of this inconsistency in the results.

In the present study BMD estimation was done using Pronosco $X$ - posure system ${ }^{\mathrm{TM}}$. Each radiograph covers the non-dominant hand and forearm. In 1999, the US Food and Drug Administration approved DXR as a clinical method for estimating BMD substantially equivalent to DXA. Due to the availability and efficacy as good as conventional and frequently used aids to measure BMD, Pronosco X-posure System was used in the present study to assess systemic BMD.

The present study is very similar in study design by Tezal et al. (5) who conducted the study on 70 postmenopausal women after adjusting for the known confounding variables. However, they found that ABL was significantly correlated with femoral BMD. A possible explanation could be the mean age of their study population, which was relatively higher than ours (62.10 vs. 57.7 yrs respectively). Secondly, the mean pack years of smoking in their study was 7.3 , whereas, in the present study only one out of 80 study subjects was current smoker; this could be the reason of significant ABL in their study as smoking has a confounding effect on alveolar bone loss. Thirdly, in their study they assessed $\mathrm{BMD}$ at spine and femur, which are weight bearing sites; whereas, in our study, BMD was assessed at non-weight bearing site (distal radius, ulna, and metacarpals) which is less likely to be influenced by body weight and physical activity.

However, the present study has the following limitations: 1) Only one subject was a smoker, so the effect of smoking on systemic bone loss and periodontitis cannot be clearly stated, 2) we did not take into account the level of education and socioeconomic status of the study population that may influence both, the periodontal disease level and skeletal BMD, 3) the cross-sectional design of our study limits the periodontal assessment to prevalent attachment loss, which is not as sensitive as measuring change in attachment level in a longitudinal study design, 4) the present study does not include any measures of oral bone densities, thus limiting any possible correlation between systemic and oral bone density. Within the limits of the present study, the following conclusions can be drawn: 1) After univariate regression analysis, only age, BMI and years since menopause affect BMD, after multivariate regression analysis, of all the variables, only BMI affects BMD; 2) Clinical attachment loss and alveolar bone loss show statistically non-significant and mildly negative correlation with the BMD; 3) CAL and ABL, though correlated to BMD are not strong predictors of BMD; 4) Systemic bone loss may be a risk indicator for periodontal destruction. To summarize, skeletal BMD is related to interproximal $\mathrm{ABL}$ and $\mathrm{CAL}$, though not to a statistically significant level; implicating postmenopausal osteopenia as a risk indicator for periodontal disease. Further studies to better understand the underlying biologic mechanisms that may link systemic bone loss and periodontal disease may provide more insights into their inter-relationship. The authors wish to thank all the subjects who participated in the study. The authors also extend their gratitude to Dr. Raghuwanshi, at Dr. Raghuwanshi's Diagnostic Center, Goa, for performing the Bone mineral density scans for all the study subjects.

\section{References}

References with links to Crossref - DOI

1. NIH Consensus Development Panel on Osteoporosis Prevention, Diagnosis, and Therapy. Osteoporosis prevention, diagnosis, and therapy. JAMA. 2001;285:785-95.

2. Assessment of fracture risk and its application to screening for postmenopausal osteoporosis. Report of a WHO Study Group. World Health Organ Tech Rep Ser. 1994;843:1-129.

3. Von Wowern N, Klausen B, Kollerup G. Osteoporosis: a risk factor in periodontal disease. J Periodontol. 1994;65:1134-8.

4. Mohammad AR, Bauer RL, Yeh CK. Spinal bone density and tooth loss in a cohort of postmenopausal women. Int J Prosthodont. 1997;10:381-5.

5. Tezal M, Wactawski-Wende J, Grossi SG, Ho AW, Dunford R, Genco RJ. The relationship between bone mineral density and periodontitis in postmenopausal women. J Periodontol. 2000;71:1492-8.

6. Inagaki K, Kurosu Y, Yoshinari N, Noguchi T, Krall EA, Garcia RI. Efficacy of periodontal disease and tooth loss to screen for low bone mineral density in Japanese women. Calcif Tissue Int. 2005;77:9-14.

7. Elders PJ, Habets LL, Netelenbos JC, van der Linden LW, van der Stelt PF. The relation between periodontitis and systemic bone mass in women between 46 and 55 years of age. J Clin Periodontol. 1992;19:492-6.

8. Klemetti E, Collin HL, Forss H, Markkanen H, Lassila V. Mineral status of skeleton and advanced periodontal disease. J Clin Periodontol. $1994 ; 21: 184-8$

9. Silness J, Loe H. Periodontal disease in pregnancy. II. Correlation between Oral hygiene and periodontal condtion. Acta Odontol Scand. 1964;22:121-35.

10. Loe H, Silness J. Periodontal disease in pregnancy. I. Prevalence and Severity. Acta Odontol Scand. 1963;21:533-51.

11. López-Marcos JF, García-Valle S, García-Iglesias AA. Periodontal aspects in menopausal women undergoing hormone replacement therapy. Med Oral Patol Oral Cir Bucal. 2005;10:132-41.

12. Vico L, Prallet B, Chappard D, Pallot-Prades B, Pupier R, Alexandre C. Contributions of chronological age, age at menarche and menopause and of anthropometric parameters to axial and peripheral bone densities. Osteoporos Int. 1992;2:153-8.

13. Hannan MT, Felson DT, Dawson-Hughes B, Tucker KL, Cupples LA, Wilson PW, et al. Risk factors for longitudinal bone loss in elderly men and women: the Framingham Osteoporosis Study. J Bone Miner Res. 2000;15:710-20.

14. Weyant RJ, Pearlstein ME, Churak AP, Forrest K, Famili P, Cauley JA. The association between osteopenia and periodontal attachment loss in older women. J Periodontol. 1999;70:982-91.

15. Taguchi A, Suei Y, Ohtsuka M, Nakamoto T, Lee K, Sanada M, et al. Relationship between self-reported periodontal status and skeletal bone mineral density in Japanese postmenopausal women. Menopause. 2005;12:144-8

16. Kribbs PJ. Comparison of mandibular bone in normal and osteoporotic women. J Prosthet Dent. 1990;63:218-22. 
17. Aström J, Bäckström C, Thidevall G. Tooth loss and hip fractures in the elderly. J Bone Joint Surg Br. 1990;72:324-5.

18. Pilgram TK, Hildebolt CF, Dotson M, Cohen SC, Hauser JF, Kardaris E, et al. Relationships between clinical attachment level and spine and hip bone mineral density: data from healthy postmenopausal women. J Periodontol. 2002;73:298-301.

19. Mohammad AR, Brunsvold M, Bauer R. The strength of association between systemic postmenopausal osteoporosis and periodontal disease. Int J Prosthodont. 1996;9:479-83.

20. Gomes-Filho IS, Passos Jde S, Cruz SS, Vianna MI, Cerqueira Ede M, Oliveira DC, et al. The association between postmenopausal osteoporosis and periodontal disease. J Periodontol. 2007;78:173140. 